\title{
Dexmedetomidine As an Adjuvant to Bupivacaine in Ultrasound Fascia Iliaca Compartment Block in Proximal End Femur Surgeries
}

\section{Hazem El-Sayed Moawad*, Abdel-Aziz A. Motawea, Ibrahim M. Elnemr, Mohamed A. Elmorshedi}

Department of Anesthesia and Surgical Intensive Care, Faculty of medicine - Mansoura University

*Corresponding author: Hazem El Sayed Moawad Weheba, Mobile: +201121516041 (+20) 01012805817,

E-Mail: hazemmoawad@yahoo.com - hazemsayed@mans.edu.eg

\begin{abstract}
Background: Fracture-neck femur is a common cause of hospital admission among the elderly population. Many patients admitted with fracture femur have long-standing cardiac, hepatic or renal problems. This makes a challenge to balance adequate analgesia with side effects of opioids. Fascia iliaca compartment block (FICB) is one of the peripheral nerve block techniques. It became widely used in providing postoperative analgesia for patient with fracture neck femur either in emergency department or in the operating room.

Objective: To evaluate the efficacy of addition of dexmedetomidine to bupivacaine on the duration and quality of postoperative analgesia in ultrasound guided fascia iliaca compartment block in proximal end femur surgeries.

Patients and methods: Sixty patients with American Society of Anesthesiologists (ASA) physical status I - II of both sexes aged from 20-60 years scheduled for proximal end femur surgeries. They were randomly assigned to one of two equal groups ( $\mathrm{n}=30$ each), using closed envelope technique: Bupivacaine group (B group), and Bupivacaine + dexmedetomidine (BD group). Result: Our study demonstrated prolongation of postoperative analgesia in bupivacaine-dexmedetomidine group (BD) compared to bupivacaine group (B). It showed statistically significant reduction in cumulative pethidine doses and prolongation in the time till first rescue analgesic is required in the $\mathrm{BD}$ group in comparison with the B group in the first 24 hours. Hemodynamic changes and incidence of side effects, were statistically insignificant among the two groups. Conclusion: Addition of dexmedetomidine, as an adjuvant to the local anesthetic bupivacaine, in ultrasound fascia iliaca compartment block provides prolongation of the duration of postoperative analgesia with less opioid consumption without remarkable side effects.
\end{abstract}

Keywords: Dexmedetomidine, Bupivacaine, Ultrasound-guided fascia iliaca, Proximal end femur surgeries.

\section{INTRODUCTION}

Lower extremities fractures are common injuries, which are associated with severe pain ${ }^{(1)}$. Positioning for neuraxial blocks is always a challenge, because even slight overriding of the fracture ends is intensely painful. Hence, prior to neuraxial blockade, analgesia is provided by conventional modes of pain relief like non-steroidal anti-inflammatory drugs (NSAIDs), opioids and also by peripheral nerve blocks such as, femoral nerve block, and fascia iliaca compartment block (FICB) ${ }^{(2)}$.

Significant postoperative pain and other morbidities almost always complicate orthopedic surgeries. Despite the publication of multiple clinical practice guidelines for pain management throughout the last decade, effective analgesia remains a significant health care concern ${ }^{(3)}$. Regional anesthesia is now proved as the best modality used by the anesthesiologists to face postoperative pain. It improves surgical outcome, reduces blood loss and furthermore reduces the postoperative morbidity ${ }^{(4)}$. Regional nerve blocks have increased popularity in the last few years with acceptance from both the surgeon and patient ${ }^{(5)}$.

(FICB) is an anterior approach to the lumbar plexus and was first described in 1989 and performed initially on children and later on adults. It was mainly used to provide analgesia following surgical procedures in the hip, femur and knee ${ }^{(\boldsymbol{6})}$. There is evidence that peripheral nerve blocks performed by ultrasound guidance are superior in terms of improved sensory and motor block, reduced need for analgesic supplements with fewer minor complications ${ }^{(7)}$. The use of ultrasound to perform the fascia iliaca block was found to be superior when compared with the traditional approach using, loss-of- resistance ee to identify the correct plane, but still requires high volumes of local anesthetic ${ }^{(8)}$.

Many additives to local anesthetics such as opioids, clonidine, neostigmine and tramadol have been used to increase the duration of the block, improve postoperative pain management and avoid the need for placing catheter for continuous local anesthetic drug infusion ${ }^{(9)}$.

Dexmedetomidine is currently in focus for its sedative, anxiolytic and analgesic properties. Pre- and intra-operative intravenous dexmedetomidine administration has shown to prolong the duration of sensory block with local anesthetics during peripheral nerve blocks ${ }^{(\mathbf{1 0})}$. Many recent studies have suggested that the addition of dexmedetomidine as adjuvant to local anesthetics shortens the sensory and motor block onset time, prolongs both sensory and motor block duration (11). It also significantly delays the first demand for analgesic supplementation, decreases $24 \mathrm{~h}$ analgesic consumption and is not associated with any major side-effect ${ }^{(\mathbf{1 2})}$.

The aim of this study was to evaluate the 
efficacy of addition of dexmedetomidine to bupivacaine on the duration and quality of postoperative analgesia in ultrasound guided fascia iliaca compartment block in proximal end femur surgeries.

\section{PATIENTS AND METHODS}

This randomized controlled blind study was conducted at Mansoura Emergency Hospital between January, 2017 and August, 2019. Sixty patients scheduled for proximal end femur surgeries, of both sexes, aged between 20 to 60 years, with ASA physical status I-II, were Included in this study.

\section{Ethical approval:}

After approval of the Institutional Review Board (IRB) at Faculty of Medicine, Mansoura University under code number (MS/16.08.12), a written informed consent was obtained from every patient before allocated in this study. This work has been carried out in accordance with The Code of Ethics of the World Medical Association (Declaration of Helsinki) for studies involving humans.

Exclusion Criteria: Exclusion criteria included Patient's refusal, ASA status III-IV, BMI > 35, Patients with multi-trauma or fractures, patients on chronic opioid use or addiction, contraindication to neuraxial anesthesia (e.g., coagulopathy, infection, etc.), infection at the site of block, previous femoral bypass surgery or inguinal hernia and history of allergy to local anesthetics or dexmedetomidine.

\section{Randomization:}

Patients were randomly allocated by closed envelope method according to medication given in FICB into 2 equal groups: ( $\mathrm{n}=30$ patients each)

Bupivacaine Group (Group B) (30 patients): $40 \mathrm{ml}$ of $0.25 \%$ bupivacaine was used in ultrasound guided FICB.

Dexmedetomidine bupivacaine Group (Group BD) (30 patients): $40 \mathrm{ml}$ of $0.25 \%$ Bupivacaine mixed with $0.5 \mathrm{mcg} / \mathrm{kg}$ dexmedetomidine was used in ultrasound guided FICB.

Blinding was achieved through the use of equal amounts of injectates used for each block in identical syringes prepared by a staff member who was not involved in the study. All blocks were performed by trained anesthetists who participated in the study.

\section{Patient preparation:}

All patients with planned proximal end femur surgeries were assessed preoperatively by detailed medical history taking, physical examination and basal laboratory investigations as complete blood count (CBC), coagulation profile, serum creatinine, liver function tests and blood sugar. Preoperatively patients were familiarized with the use of Visual Analogue scale (VAS) for pain assessment, where "0"no pain and " 10 " the most severe pain.

\section{Intra-operative management:}

In the operating theater, routine monitoring was applied including electrocardiography (ECG), noninvasive mean arterial pressure (MAP) and peripheral oxygen saturation $\left(\mathrm{SPO}_{2}\right)$. All patients were cannulated for fluid infusion and FICB was done half an hour before spinal anesthesia.

Technique of the ultrasound guided fascia iliaca compartment block:

This block was performed with the patient in the supine position and the bed flattened to maximize access to the inguinal area. We used Korean Siemens ACUSON X300 ultrasound device in all patients. We started by sterilization of the area of block by antiseptic solution then injection of 1-2 $\mathrm{ml}$ of $1 \%$ lidocaine as a local anesthetic. For the approach below the inguinal ligament, we used a high frequency ultrasound probe $(13-16 \mathrm{MHz})$ in a transverse direction below the inguinal ligament and identified the femoral artery pulsation and the iliacus muscle. Under complete aseptic technique, the needle (22gauge spinal needle) was inserted in plane of the ultrasound beam, below the fascia iliaca and injection of local anesthetic (40 $\mathrm{ml}$ of the study solution) was done.

\section{Assessment of FICB:}

Femoral, obturator and lateral femoral cutaneous nerve dermatomes was assessed for sensory block with ice-cold test every $5 \mathrm{~min}$ for $30 \mathrm{~min}$ after the FICB at all patients. We assessed nerves that had been blocked at upper medial thigh for obturator nerve, anterior thigh for femoral nerve and lateral part of the thigh for lateral femoral cutaneous nerve.

\section{Spinal anesthesia:}

Under complete aseptic technique spinal anesthesia was performed in the sitting position at L34 intervertebral space. $15 \mathrm{mg}$ hyperbaric bupivacaine $0.5 \%$ was injected in the subarachnoid space using a 25 -gauge spinal needle.

\section{Postoperative Assessment:}

At the end of surgery, patients were transferred to the post anesthetic care unit (PACU). The VAS score was being assessed after surgery and whenever VAS $\geq 4$, postoperative analgesia was provided with slow intravenous (IV) $25 \mathrm{mg}$ pethidine as a single dose. During the following 4 hours after pethidine injection, if VAS still $\geq 4$ slow IV $30 \mathrm{mg}$ ketorolac was given.

Sensory and motor block of spinal anesthesia were evaluated in the non-traumatized limb in the PACU. The sensory block duration (the time from intrathecal injection till sensory recovery at the level of S1) was assessed by the return of the pin-prick sensation on lateral aspect of the foot. The duration of motor block (the time from of intrathecal injection to complete regression of motor block) was assessed by ability to lift the extended leg using Bromage scale. 


\section{Recorded Data:}

VAS was recorded basally (before injection of FICB), 10 minutes after the injection, prior to spinal anesthesia, 30 min after the injection, 1hour, $2 \mathrm{~h}, 3 \mathrm{~h}, 4 \mathrm{~h}, 6 \mathrm{~h}, 8 \mathrm{~h}, 12 \mathrm{~h}$, $18 \mathrm{~h}$, till 24 hours after the end of surgery. Hemodynamic data (Heart rate, mean arterial blood pressure (MAP)) were recorded at the same times of pain assessment. Level of spinal anesthesia, onset \& duration of motor and sensory blocks, duration of surgery, time till the first rescue analgesic, total analgesic requirements in the first 24 hours after surgery, and any complication related to the drugs were recorded.

\section{Sample size:}

G power program (3.3.9.2) was used to calculate sample size. The time to the first request for analgesia used as the primary effect. One tailed t test for difference between two independent means was the computed statistical test. Effect size was chosen as $0.4, \alpha$ error was 0.05 and a study power (1-ß error) of 0.9 was used. The resulted sample size was 30 patients for each group.

\section{Statistical analysis}

The collected data were coded, processed and analyzed using the SPSS (Statistical Package for Social Sciences) version 22 for Windows ${ }^{\circledR}$ (IBM SPSS Inc., Chicago, IL, USA). Data were tested for normal distribution using the Shapiro Walk test. Qualitative data were represented as frequencies and relative percentages. Chi square test $\left(\chi^{2}\right)$ to calculate difference between two or more groups of qualitative variables. Quantitative data were expressed as mean \pm SD. Independent samples t-test was used to compare between two independent groups of normally distributed variables (parametric data). $\mathrm{P}$ value $\leq 0.05$ was considered significant.

\section{RESULTS}

Patient's demographic data showed no statistically significant differences regarding age, sex and body mass index (BMI) among the two studied groups (Table 1).

The intraoperative HR and MBP showed statistically insignificant differences among the two studied groups (Table $2 \& 3$ ).

Motor and sensory block duration of spinal anesthesia was statistically insignificant among the two studied groups (Table 4).

There was a statistically significant reduction of cumulative pethidine doses, while the time to the first request for analgesic in the $\mathrm{BD}$ group was longer in comparison with the B group in the first 24 hours (29 $\mathrm{mg}$ versus $62 \mathrm{mg}$ respectively) (Table 5).

Pain score at rest showed statistically significant decrease in the BD group at 8,12 postoperative hours as compared to the B group (Table 6).

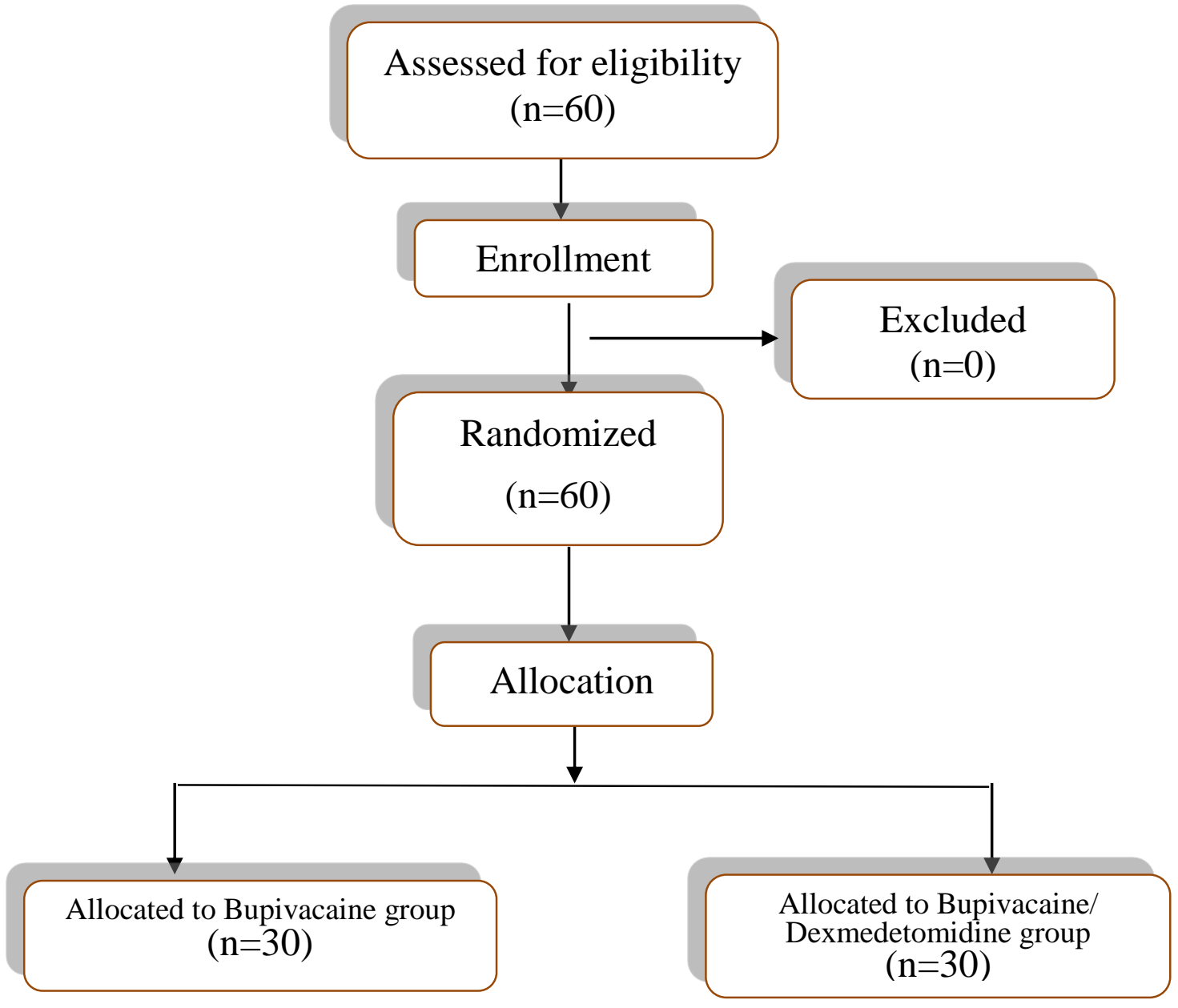

Figure (1): CONSORT flow diagram. 
Table (1): Demographic data in the studied groups

\begin{tabular}{lccc}
\hline Items & $\begin{array}{c}\text { Group (B) } \\
(\mathbf{n = 3 0})\end{array}$ & $\begin{array}{c}\text { Group (BD) } \\
(\mathbf{n = 3 0})\end{array}$ & P. value \\
\hline Age (years) & $54.10 \pm 4.27$ & $51.66 \pm 7.40$ & 0.268 \\
Sex: & $17(56.7 \%)$ & $16(53.3 \%)$ & \\
-Male & $13(43.3 \%)$ & $14(46.7 \%)$ & 0.795 \\
-Female & $22.67 \pm 3.1$ & $23.65 \pm 2.25$ & 0.160 \\
BMI (kg/m ${ }^{2}$ ) & $20 / 10$ & $19 / 11$ & 0.787 \\
Type of surgery (DHS/Bipolar) & $115.9 \pm 25.4$ & $124.2 \pm 34.5$ & 0.294 \\
Duration of surgery (minutes) & $10(33.33 \%)$ & $12(40 \%)$ & 0.256 \\
& $20(66.67 \%)$ & $18(60 \%)$ &
\end{tabular}

Data were expressed as Mean \pm Standard deviation, except for Sex; expressed as Number \pm Percentage

B: Bupivacaine, BD: bupivacaine \& dexmedetomidine, $\quad \mathrm{n}$; number of cases $\quad$ BMI: Body mass index,

kg: kilogram, cm: centimeter, DHS: dynamic hip screw P-value is considered significant if calculated $<0.05$

Table (2): Heart rate (beats $/ \mathrm{min}$ ) of the study in both groups. Data are in mean \pm SD

\begin{tabular}{cccc}
\hline Time & $\begin{array}{c}\text { Group }(\mathbf{B}) \\
(\mathbf{n = 3 0})\end{array}$ & $\begin{array}{c}\text { Group (BD) } \\
(\mathbf{n = 3 0})\end{array}$ & P. value \\
\hline Basal (before FICB) & $106.7 \pm 6.7$ & $107.3 \pm 8.3$ & 0.742
\end{tabular}

After 10 min from FICB

$99.13 \pm 5.2 \quad 100.23 \pm 5.8 \quad 0.441$

After 20 min from FICB

$\begin{array}{lll}95.3 \pm 8.3 & 94.0 \pm 9.0 & 0.560\end{array}$

After 30 min from FICB
$92.21 \pm 3.3$
$91.06 \pm 4.1$
0.300

After 1h. from FICB (30min.

after spinal anesthesia)

$71.09 \pm 3.4$

$71.28 \pm 3.5$

0.827

After 2h. from FICB

$78.33 \pm 10.9$

$78.86 \pm 7.6$

0.745

After 4h. from FICB

$79.3 \pm 8.0$

$82.2 \pm 7.4$

0.147

After $\mathbf{6 h}$. from FICB

$82.1 \pm 7.5$

$81.4 \pm 9.1$

0.751

After 8h. from FICB

$81.89 \pm 7.8$

$81.5 \pm 8.6$

0.856

After 12. from FICB

$82.3 \pm 8.9$

$79.7 \pm 9.6$

0.281

After 18h. from FICB

$82.0 \pm 9.0$

$80.1 \pm 8.5$

0.380

After 24h. from FICB

$82.2 \pm 7.4$

$79.3 \pm 8.0$

0.147

\footnotetext{
*P-value <0.05: statistically significant.
} 
Table (3): Mean arterial blood pressure ( $\mathrm{mmHg}$ ) values of the study in both groups (Data are in mean $\pm \mathrm{SD}$ ).

\begin{tabular}{lccc}
\hline Time & $\underset{(n=30)}{\text { Group (B) }}$ & $\begin{array}{c}\text { Group (BD) } \\
(\mathbf{n}=30)\end{array}$ & P. value
\end{tabular}

Basal (Before FICB)

After 10 min from

FICB

After $\mathbf{2 0}$ min from

FICB

After 30 min from

FICB

After 1h. from FICB

(30min. after spinal anesthesia)

After 2h. from FICB

After 4h. from FICB

After $\mathbf{6 h}$. from FICB

After 8h. from FICB

After 12h. from FICB

After 18h. from FICB

After 24h. from FICB

$92.62 \pm 6.4$

$91.06 \pm 4.1$

$89.29 \pm 7.8$

$84.81 \pm 5.2$

$64.53 \pm 8.4$

$74.15 \pm 8.3$

$87.83 \pm 8.2$

$85.83 \pm 8.8$

$84.13 \pm 8.9$

$86.40 \pm 8.8$

$86.40 \pm 9.0$

$87.34 \pm 7.8$
$92.84 \pm 6.8$

0.895

$92.21 \pm 3.34$

0.300

$86.7 \pm 9.0$

0.241

$83.62 \pm 5.6$

0.384

$65.96 \pm 6.5$

0.468

$73.70 \pm 7.7$

0.792

$84.10 \pm 6.5$

0.245

$84.53 \pm 6.5$

0.399

$84.33 \pm 6.8$

0.289

$84.27 \pm 6.3$

0.468

$84.27 \pm 6.5$

0.354

$85.18 \pm 7.5$

0.365

*P-value <0.05: statistically significant

Table (4): Motor and sensory block duration (hour) of spinal anesthesia

\begin{tabular}{lccc}
\hline \multicolumn{1}{c}{ Items } & $\begin{array}{l}\text { Group }(\mathbf{B}) \\
(\mathbf{n = 3 0 )}\end{array}$ & $\begin{array}{l}\text { Group (BD) } \\
(\mathbf{n = 3 0})\end{array}$ & P. value \\
\hline $\begin{array}{l}\text { Duration of sensory block } \\
\text { (hours) }\end{array}$ & $3.3 \pm 0.7$ & $3.15 \pm 0.81$ & $\mathbf{0 . 4 1 2}$ \\
$\begin{array}{l}\text { Duration of motor block } \\
\text { (hours) }\end{array}$ & $2.41 \pm 0.56$ & $2.39 \pm 0.61$ & $\mathbf{0 . 7 1 2}$ \\
\hline
\end{tabular}

Data expressed as Mean \pm Standard deviation B: Bupivacaine, D: bupivacaine $\&$ dexmedetomidine, n: number, $\mathrm{P}$-value is significant if calculated $<0.05 * \&$ highly significant if calculated $<0.001 * *$ 
Table (5): The time to the first request for analgesia (hours), total pethidine (mg) requirement in the first 24 hours, and number of patients required additional analgesia (ketorolac $30 \mathrm{mg}$ ) in the studied groups

$\begin{array}{lccc}\text { Items } & \begin{array}{c}\text { Group (B) } \\ (\mathbf{n}=30)\end{array} & \begin{array}{c}\text { Group (BD) } \\ (\mathbf{n}=\mathbf{3 0})\end{array} & \text { P. value }\end{array}$

\section{The time to the first request for analgesia (hours)}

Pethidine (mg)

Number of patients required

(ketorolac $30 \mathrm{mg}$ )

$$
7.43 \pm 2.12
$$

$15.2 \pm 4.81^{*}$

$<0.001$

$62 \pm 17.5$

$29 \pm 11.5^{*}$

$<0.001$

$15(50 \%)$

$6(20 \%)^{*}$

0.014

Data expressed as Mean \pm Standard deviation, B: Bupivacaine, BD: bupivacaine $\&$ dexmedetomidine, $\mu \mathrm{g}$ : microgram, n: number, P-value is significant if calculated $<0.05 *$

Table (6): Perioperative assessment of visual analogue scale (VAS) score (from 1-10) Data are in median (IQR)

$\begin{array}{cccc}\text { Group }(\mathbf{B}) & \text { Group (BD) } & (\mathbf{n}=\mathbf{3 0}) & \text { P. value }\end{array}$

Basal (Before FICB)

After 10 min from FICB

After 20 min from FICB

After 30 min from FICB

$2(2-4)$

2(2-3)

0.563

After 1h. from FICB

(30min. after spinal

anesthesia)

After $\mathbf{2 h}$. from FICB

$1(1-2)$

$1(1-2)$

0.563

After 4h. from FICB

$2(1-2)$

$1(1-2)$

0.100

After $\mathbf{6 h}$. from FICB

$2(1-3)$

$2(1-2)$

0.299
After 8h. from FICB

$4(3-5)$

$4(3-6)$

$4(3-6)$

$4(3-5)$

$2(1-3) *$

$<0.001$

After12h. from FICB

After18h. from FICB

After24h. from FICB
$3(2-3) *$

$<0.001$

$4(3-5)$

0.518

$4(3-5)$
0.876

$\mathrm{P}$-value is significant if calculated $<0.05 *$ 


\section{DISCUSSION}

This randomized study was designed to evaluate the safety and efficacy of dexmedetomidine administered as an adjunct to the local anesthetic bupivacaine for postoperative analgesia in patients undergoing proximal end femur surgeries. The result of our study demonstrated prolongation of postoperative analgesia in bupivacaine-dexmedetomidine group (BD) in comparison with bupivacaine group (B). It showed statistically significant reduction in cumulative pethidine doses and prolongation of the time till first call for analgesia in the BD group in comparison with the B group in the first postoperative 24 hours. VAS score was statistically significant at 8,12 postoperative hours in the $\mathrm{BD}$ group in comparison with the $\mathrm{B}$ group.

In regard to hemodynamic changes and incidence of side effects, differences were statistically insignificant among the two groups.

Many studies had confirmed that FICB has an analgesic effect after hip surgery, Goitia-Arrola $\boldsymbol{e t}$ al. (13) found that fascia iliaca compartment block was effective in controlling initial postoperative pain in the first few hours after total hip surgery. Also Krych et al. (16) reported that FICB decreased opioid consumption, and provided a high quality of pain relief and patient satisfaction after hip surgery. On the other hand, Shariat et al. ${ }^{(14)}$ reported no significant difference in postoperative pain score and 24 hours opioid consumption in 32 patients receiving FICB after total hip replacement. This may be due to low volume injected (30ml.). Helayel et al. ${ }^{(15)}$ showed that the effective volumes of local anesthetics in the FICB capable of producing a block in $99 \%$ of cases were 37.3 $\mathrm{mL}$ for bupivacaine and $36.6 \mathrm{~mL}$ for ropivacaine.

Dexmedetomidine produces differential sensory - motor blockade (more sensory), which is unlikely to be achieved with dexamethasone ${ }^{(19)}$ and often occur with liposomal bupivacaine ${ }^{(3)}$. Abdallah et al. ${ }^{(18)}$ reported that dexmedetomidine has the advantage of prolongation of the duration of local anaesthetics. Brummett et al. (17) showed that dexmedetomidine prolonged the duration of sciatic nerve block after experimental and clinical studies.

As regards, the time till first rescue analgesia and total opioid consumption, Hua et al. ${ }^{(20)}$ found that using dexmedetomidine combined with ropivacaine in FICB, provided prolongation of time till first rescue analgesia with less total opioid consumption and low level of VAS, which cope with our results. Prabha et al. (21) demonstrated that dexmedetomidine combined with ropivacaine prolongs the duration of postoperative analgesia in TAP block (with less postoperative visual analogue score) and reduces the postoperative analgesic requirements post hernia repair. Parameswari and Udayakumar (22) reported that TAP block after Caesarean section using dexmedetomidine as an adjuvant to bupivacaine, lead to prolongation of the time till first dose of rescue analgesia and reduction of the total dose of opioid requirement in the first 24-h. Dexmedetomidine at dose of $1 \mathrm{mcg} / \mathrm{kg}$ produces a high quality of peripheral nerve block with minimal side effects ${ }^{(23)}$.

In the current study, we used dexmedetomidine in a dose of $0.5 \mathrm{mcg} / \mathrm{kg}$ and didn't find statistical significance between the two groups as regards side effects of dexmedetomidine. Similar to our study, Hua et al. ${ }^{(20)}$ found that FICB using dexmedetomidine with ropivacaine didn't affect the patient's cardiovascular indices. Abdelaal et al. ${ }^{(24)}$ showed that addition of dexmedetomidine to levobupivacaine in TAP block improved analgesia in patients undergoing abdominoplasty with no remarkable side effects. In a meta-analysis, El-Boghdadly et $\boldsymbol{a l} .{ }^{(25)}$ concluded that dexmedetomidine injection in supraclavicular brachial plexus block produces more prolongation in the duration of sensory and motor block, with prolonged postoperative analgesia. The benefits of dexmedetomidine outweighed the increased risk of transient bradycardia. Patro et al. (26) showed that addition of dexmedetomidine to intrathecal bupivacaine in infraumblical surgeries lead to longer duration of anaesthesia and analgesia with haemodynamic stability as compared to bupivacaine alone.

\section{CONCLUSION}

Addition of dexmedetomidine, as an adjuvant to the local anesthetic bupivacaine, in ultrasound fascia iliaca compartment block provides prolongation of the duration of postoperative analgesia with less opioid consumption without remarkable side effects.

\section{Financial support and sponsorship: Nil. Conflict of interest: Nil.}

\section{REFERENCES}

1. Okereke I, Abdelmonem M (2021): Fascia Iliaca Compartment Block for Hip Fractures: Improving Clinical Practice by Audit. Cureus, 13 (9): e17836.

2. Gopal N, Krishnamurthy D (2018): A Clinical Comparative Study of Fascia Iliaca Compartment Block with Bupivacaine and Bupivacaine with Dexmedetomidine for Positioning and Duration of Postoperative Analgesia in Fracture Femur under Spinal Anesthesia. Anesth Essays Res., 12 (2): 528534.

3. Pinson $\mathbf{S}$ (2015): Fascia Iliaca (FICB) block in the emergency department for adults with neck of femur fractures: A review of the literature. International Emergency Nursing, 23 (4): 323-328.

4. Chen Y, Boden K, Schreiber K (2021): The role of regional anaesthesia and multimodal analgesia in the prevention of chronic postoperative pain: a narrative review. Anaesthesia, 76 (1): 8-17.

5. Miller G, Godrey J, Sagmeister M et al. (2016): Provision of fascia iliaca compartment block in the acute management of proximal femoral fractures: A national observational study of UK hospitals. Injury, 47 (11): 2490-2494. 
6. Groot L, Dijksman L, Simons M et al. (2015): Single Fascia Iliaca Compartment Block is safe and Effective for Emergency Pain Relief in Hip-fracture Patients. Western Journal of Emergency Medicine, 16 (7): 1188 1193.

7. Lewis S, Price A, Walker K et al. (2015): Ultrasound guidance for upper and lower limb blocks. Cochrane Database of Systematic Reviews. Pp: 1-110. https://www.journalslibrary.

nihr.ac.uk/downloads/other-nihr-research/cochraneprogramme-grants/Ultrasound-guidance-for-upperand-lower-limb-blocks.pdf

8. Dolan J, Williams A, Murney E et al. (2008): Ultrasound guided fascia iliaca block: a comparison with the loss of resistance technique. Regional Anesthesia and Pain Medicine, 33 (6): 526-531.

9. Vadhanan P, Tripaty D, Adinarayanan S (2015): Physiological and pharmacologic aspects of peripheral nerve blocks. Journal of Anaesthesiology, Clinical Pharmacology, 31 (3): 384-393.

10. Abdelhamid A, Abdelhaq M, Yasin M et al. (2016): Dexmedetomidine as an additive to local anesthetics compared with intravenous dexmedetomidine in peribulbar block for cataract surgery. Saudi Journal of Anaesthesia, 10 (1): 50-54.

11. Kaur H, Singh G, Rani S et al. (2015): Effect of dexmedetomidine as an adjuvant to levobupivacaine in supraclavicular brachial plexus block: A randomized double-blind prospective study. Journal of Anaesthesiology Clinical Pharmacology, 31 (3): 33337.

12.Abdallah F, Dwyer C, Ogilvie-Harris N (2016): IV and Perineural Dexmedetomidine Similarly Prolong the Duration of Analgesia after Interscalene Brachial Plexus Block: A Randomized, Three-arm, Triplemasked, Placebo-controlled Trial. Anesthesiology, 124 (3): 683-695.

13. Goitia-Arrola L, Telletxea $S$, Martínez Bourio $\mathbf{R}$ et al. (2009): Fascia iliaca compartment block for analgesia following total hip replacement surgery. Revista Espanola de Anestesiologia Y Reanimacion, 56 (6): $343-348$.

14. Krych A, Baran S, Kuzma S et al. (2014): Utility of multimodal analgesia with fascia iliaca blockade for acute pain management following hip arthroscopy. Knee Surgery, Sports Traumatology, Arthroscopy, 22 (4): 843-847.

15.Shariat A, Hadzic A, Xu D et al. (2013): Fascia lliaca Block for Analgesia after Hip Arthroplasty. Regional Anesthesia and Pain Medicine, 38 (3): 201- 205.

16. Helayel $P$, Lobo G, Vergara $R$ et al. (2006): Effective volume of local anesthetics for fascia iliac compartment block: a double-blind, comparative study between $0.5 \%$ ropivacaine and $0.5 \%$ bupivacaine. Revista Brasileira de Anestesiologia, 56 (5): 454-460.
17. Abdallah F, Johnson J, Chan V et al. (2015): Intravenous dexamethasone and perineural dexamethasone similarly prolong the duration of analgesia after supraclavicular brachial plexus block: A randomized, triple-arm, double-blind, placebocontrolled trial. Regional Anesthesia and Pain Medicine, 40 (2): 125-132.

18. Abdallah F, Abrishami A, Brull R (2013): The Facilitatory Effects of Intravenous Dexmedetomidine on the Duration of Spinal Anesthesia. Anesthesia \& Analgesia, 117 (1): 271-278.

19. Brummett C, Norat M, Palmisano J et al. (2008): Perineural Administration of Dexmedetomidine in Combination with Bupivacaine Enhances Sensory and Motor Blockade in Sciatic Nerve Block without Inducing Neurotoxicity in Rat. Anesthesiology, 109 (3): 502-511.

20.Hua X, Hu Y, Chen D et al. (2017): Efficacy and safety of ultrasound-guided fascia iliaca compartment block using dexmedetomidine combined with ropivacaine in aged patients undergoing hip replacement. Int J Clin Exp Med., 10 (12): 1648416491.

21.Prabha R, Raman R, Kumar M (2015): 2015 Effect of Adding Dexmedetomidine to Ropivacaine for Transversus. Journal of Medical Science and Clinical Research, 3 (2): 4550-4557.

22.Parameswari R, Udayakumar P (2017): Comparison of Efficacy of Bupivacaine with Dexmedetomidine Versus Bupivacaine Alone for Transversus Abdominis Plane Block for Post-operative Analgesia in Patients Undergoing Elective Caesarean Section. The Journal of Obstetrics and Gynecology of India, 68 (2): 98-103.

23.Hariharan U, Natarajan V (2017): Dexmeditomidine and Anaesthesia: Indications and Review of Literature Dexmeditomidine and Anaesthesia: Indications and Review of Literature. International Journal of Clinical Anesthesiology, 5(4): 1079.

24. Abdelaal W, Metry A, Refaat M et al. (2015): Comparative Study between Levobupivacine versus Levobupivacaine Plus Dexmedetomidine for Transversus Abdominis Plane Block "TAP" in PostOperative Pain Management after Abdominoplasty. Enliven: Journal of Anesthesiology and Critical Care Medicine, 2 (2): 4-9.

25.El-Boghdadly K, Brull R, Sehmbi H et al. (2017): Perineural Dexmedetomidine Is More Effective Than Clonidine When Added to Local Anesthetic for Supraclavicular Brachial Plexus Block. Anesthesia \& Analgesia, 124 (6): 2008-2020.

26.Patro S, Deshmukh H, Ramani Y et al. (2016): Evaluation of Dexmedetomidine as an Adjuvant to Intrathecal Bupivacaine in Infraumbilical Surgeries. Journal of Clinical and Diagnostic Research, 10 (3): 1316. 